OPEN ACCESS

Edited by:

José Bines,

Instituto Nacional de Câncer (INCA), Brazil

Reviewed by:

Vidya Sethunath

Baylor College of Medicine,

United States

Martin Hernán Bonamino,

Instituto Nacional de Câncer

(INCA), Brazil

*Correspondence:

Xiu Nie

niexiuyishi@126.com

Jing Cheng

chenjin1118@hotmail.com

tThese authors share first authorship

Specialty section:

This article was submitted to

Women's Cancer,

a section of the journal

Frontiers in Oncology

Received: 21 September 2019

Accepted: 20 December 2019

Published: 22 January 2020

Citation:

Wang Q, Xiang Q, Yu L, Hu T, Chen Y,

Wang J, Nie X and Cheng J (2020)

Changes in Tumor-Infiltrating

Lymphocytes and Vascular

Normalization in Breast Cancer

Patients After Neoadjuvant

Chemotherapy and Their Correlations

With DFS. Front. Oncol. 9:1545.

doi: 10.3389/fonc.2019.01545

\section{Changes in Tumor-Infiltrating Lymphocytes and Vascular Normalization in Breast Cancer Patients After Neoadjuvant Chemotherapy and Their Correlations With DFS}

\author{
Qiong Wang ${ }^{1 \dagger}$, Qun Xiang ${ }^{1 \dagger}$, Lan $\mathrm{Yu}^{2}$, Ting $\mathrm{Hu}^{1}$, Yangyang Chen ${ }^{1}$, Jue Wang ${ }^{1}$, Xiu Nie ${ }^{2 \star}$ \\ and Jing Cheng ${ }^{1 *}$ \\ ${ }^{1}$ Cancer Center, Union Hospital, Tongji Medical College, Huazhong University of Science and Technology, Wuhan, China, \\ ${ }^{2}$ Department of Pathology, Union Hospital, Tongji Medical College, Huazhong University of Science and Technology, Wuhan, \\ China
}

Objective: Changes in the number of various tumor-infiltrating lymphocytes (TILS) and degrees of vascular normalization in breast cancer (BC) patients after neoadjuvant chemotherapy (NAC) were analyzed to screen key factors that can predict the prognosis.

Methods: HE-stained sections were used to assess the degree of TILs infiltration; immunohistochemically stained sections were used to assess the infiltration of CD8+, CD4+, FOXP3+ Tregs and the expression of PD-L1; immunofluorescence-stained sections were used to assess the microvessel density (MVD) and microvessel pericyte coverage index (MPI). The expression of them before NAC were compared with those after NAC, and correlations between changes in these parameters and the pathological complete remission (pCR) and DFS of BC patients were analyzed.

Results: After NAC, the percentage of patients with enhanced STILs in the pCR group was significantly higher than that in the Non-pCR group $(P<0.05)$. Univariate and multivariate analyses showed that the number of FOXP3+ Tregs and MPI before NAC were correlated with $\mathrm{pCR}(P<0.05)$. Survival analysis showed that the DFS of BC patients with reduced FOXP3+ Tregs was significantly better than that of patients with elevated FOXP3+ Tregs $(P=0.029)$. The sTILs count and MPI were significantly higher in primary tumors than lymph nodes $(P<0.05)$.

Conclusion: After NAC, the reduced infiltration of FOXP3+ Tregs was correlated with an improvement in DFS in BC patients. Changes in the number of FOXP3+ Tregs and the MPI may be used as prognostic markers for BC patients.

Keywords: breast cancer, tumor-infiltrating lymphocyte, neoadjuvant chemotherapy, microvessel pericyte coverage index, FOXP3+ Tregs 


\section{INTRODUCTION}

Among females, breast cancer (BC) is the malignant tumor with the highest incidence (1) and seriously threatens the health of women worldwide (2). The incidence of BC in China increases yearly, and the mortality rate remains high (3). The primary reason for failure of $\mathrm{BC}$ treatment is relapse and metastasis of BC after treatment $(4,5)$. Neoadjuvant chemotherapy (NAC) is currently the main treatment for locally advanced $\mathrm{BC}$ and it can be used to detect the sensitivity of tumor cells to anticancer treatment and to guide the follow-up treatment (6). Our understanding of these patients is still insufficient; recurrence and metastasis still occur in patients who achieve PCR after NAC.

Tumor-infiltrating lymphocytes (TILs) are the most important immune cells in the tumor microenvironment (TME) (7). For BC, according to the distribution of TILs in tumor tissues, TILs can be divided into intratumoral TILs (iTILs), and stromal TILs (sTILs). The International TILs Working Group recommends the use of sTILs to assess TILs in BC (8). The traditional view is that BC lacks immunological activity (9). However, a growing body of evidence suggests that pretreatment of sTILs in BC patients can predict treatment response and is correlated with prognosis $(10,11)$. Therefore, sTILs may become a biomarker for efficacy prediction and the prognosis of BC patients $(12,13)$. Denkert divided BC patients into three groups according to sTILs infiltration in tumor tissues: low sTILs infiltration (0-10\%), medium sTILs infiltration (11-59\%) and high sTILs infiltration (60-100\%), their studies suggested that high sTILs infiltration can predict the NAC response of all $\mathrm{BC}$ subtypes and is correlated with the survival benefit of Her2-positive $\mathrm{BC}$ and triple-negative breast cancer (TNBC) (14). In addition to TILs, programmed death ligand 1 (PD-L1) in the TME is widely studied (15). The expression of PD-L1 is closely correlated with the presence of TILs. Programmed death-1 (PD-1) expressed by TILs binds to PD-L1 expressed on tumor cells to allow tumors to escape anti-tumor immunity in the TME of BC (16).

Another key factor in the TME of BC is vascular normalization, which involves increased pericyte coverage, improved tumor vessel perfusion, reduced vascular permeability, and consequently mitigated hypoxia (17). Vascular normalization can promote $\mathrm{T}$ lymphocyte infiltration, and interferon- $\gamma$ secreting $\mathrm{T}$ helper 1 (Th1) cells are the major cell population associated with vascular normalization, which increases pericyte coverage and tumor vessel normalization $(18,19)$. Microvessel density (MVD) is an important indicator for evaluating tumor angiogenesis (20). The microvessel pericyte coverage index (MPI) is an important indicator reflecting vascular normalization. MPI means the ratio of microvascular density covered by pericytes to total microvascular density in tumor tissues. The MPI of most tumor blood vessels is low, and the function of the tumor vascular bed is not mature (21). The currently available studies on tumor vascular normalization and TILs are primarily fundamental studies that have not been conducted in clinical practice.
In the present study, changes in sTILs, PD-L1 expression and vascular normalization were examined in $\mathrm{BC}$ patients before and after NAC, the effect of NAC on the TME of BC was investigated, and its correlation with the post-NAC pCR rate and disease-free survival (DFS) was analyzed to find appropriate predictive and prognostic biomarkers for treatment of BC.

\section{MATERIALS AND METHODS}

\section{Research Subjects}

This study included $75 \mathrm{BC}$ patients who were admitted to the Cancer Center, Union Hospital, Tongji Medical College, Huazhong University of Science and Technology, and treated with NAC between November 2013 and December 2018. All patients underwent core needle biopsy at initial diagnosis and were pathologically confirmed as invasive BC. All patients received four or more cycles of NAC, which was a combined chemotherapy using anthracyclines, cyclophosphamide, or taxanes. For patients with HER-2 positive BC, Trastuzumab was used. No other drugs were used as NAC treatment in our study including Pertuzumab and endocrine therapy etc. According to the pathological biopsy report, patients were divided into four molecular subtypes, HER2-positive BC, luminal/HER2-negative BC, luminal/HER2-positive BC, and TNBC. Three weeks after NAC, modified radical mastectomy or breast-conserving surgery for BC was performed in our hospital. After the surgery, the appropriate standard adjuvant therapy (radiotherapy, chemotherapy, targeted therapy, or endocrine therapy) was administered according to patient conditions.

(1) Inclusion criteria:

1) Female patients with preoperative biopsy-confirmed invasive $\mathrm{BC}$;

2) Patients who received 4 or more cycles of preoperative NAC;

3) Patients who underwent modified radical mastectomy or breast-conserving surgery after NAC;

4) Patients who did not have any other immune system diseases and did not use immunosuppressants.

(2) Exclusion criteria:

1) Male BC patients;

2) Patients with second primary cancer at other sites at the time of diagnosis;

3) Patients who received fewer than 4 cycles of preoperative NAC;

4) Patients with incomplete pathological and follow-up data.

\section{Collection of the Clinical Data}

The general clinical data of patients included time of diagnosis, age, diagnosis, pathological data before NAC, NAC regimens, operation time, surgical pathology data, postoperative treatment method, and post-treatment follow-up data. PCR was the primary end point of this study, and pCR was defined as follows: after NAC, no primary invasive cancer component was observed in primary tumors or metastatic lymph nodes, or only components of carcinoma in situ were observed in primary tumors and metastatic lymph nodes. DFS was the secondary end 
point of the study and was defined as the time from the date of surgery to the date of disease progression.

Paraffin-embedded specimens from the core needle biopsy before NAC and paraffin-embedded specimens from surgery were collected from the specimen bank of the Department of Pathology in our hospital. For patients who achieved pCR after NAC, paraffin-embedded specimens from the tumor bed were selected. For patients who did not achieve pCR, if the lymph nodes were negative, paraffin-embedded specimens from residual tumors were selected; if lymph nodes were positive, paraffin-embedded specimens from residual tumors and metastatic lymph nodes were both selected. Each paraffinembedded specimen was continuously sectioned into six pieces, and the thickness of each section was $4 \mu \mathrm{m}$. Hematoxylin-eosin (HE), immunohistochemical and immunofluorescence staining were performed.

\section{HE Staining, Immunohistochemistry, and Immunofluorescence}

Paraffin-embedded sections were dewaxed and hydrated, followed by hematoxylin and eosin staining, dehydration, mounting and microscopic observation, and analysis. The prepared paraffin-embedded sections were used to accept immunohistochemistry and immunofluorescence testing. The antibodies used were showed in the Table 1.

\section{Pathological Evaluation}

1. Assessment of sTILs: (1) According to the recommendations of the International TILs Working Group (8), the sTILs score was defined as the area percentage of mononuclear inflammatory cells in the tumor stroma. The sTILs score was set as a continuous variable, and the positive cutoff value was set at $1 \%$. (2) The entire HE-stained section was first observed under low magnification $(\times 10)$ to evaluate whether the distribution of sTILs was uniform. (3) If the distribution of sTILs was relatively uniform, three high-magnification $(\times 40)$ views were randomly selected to calculate the mean sTILs count. (4) If the distribution of sTILs was not uniform, the regions with the highest and lowest sTILs

TABLE 1 | Concentrations of primary antibodies.

\begin{tabular}{lll}
\hline Primary antibody & Source & Concentration \\
\hline $\begin{array}{l}\text { CD8 rabbit anti-human polyclonal } \\
\text { antibody (17335-1-AP) }\end{array}$ & Proteintech, & $1: 1,000$ \\
CD4 mouse anti-human monoclonal & DAKO, & Ready-to-use \\
antibody & Denmark & \\
FOXP3 rabbit polyclonal antibody & Proteintech, & $1: 1,600$ \\
$\begin{array}{l}\text { (22228-1-AP) } \\
\text { PD-L1 rabbit anti-human polyclonal }\end{array}$ & PS & \\
$\begin{array}{l}\text { antibody (28076-1-AP) } \\
\text { CD105 mouse anti-human }\end{array}$ & US & $1: 200$ \\
monoclonal antibody (BM1725) & Boster, & $1: 100$ \\
NG2 rabbit anti-human monoclonal & Wuhan, China & \\
Abcam, UK & $1: 100$
\end{tabular}

numbers were excluded before three high-magnification views were randomly selected to calculate the mean sTILs count.

2. Evaluation of CD8+ T cells, CD4+ T cells, and FOXP3+ Tregs: (1) First, the entire section was evaluated under low magnification $(\times 10)$ to observe whether the positive cells were uniformly distributed in tumor stroma; (2) if the distribution of positive cells was relatively uniform, three high- magnification views $(\times 40)$ were randomly selected to count the number of positive cells, and the mean positive cell count was obtained. (3) If the distribution of positive cells was not uniform, the regions with the highest and lowest numbers of positive cells were excluded before three highmagnification views were randomly selected to count the number of positive cells, and the mean positive cell count (unit: /HPF) was obtained.

TABLE 2 | Patients clinical characteristics.

\begin{tabular}{ll}
\hline Parameter & N (\%)
\end{tabular}

Age

$\begin{array}{ll}<50 \text { years } & 47(62.7) \\ \geq 50 \text { years } & 28(37.3)\end{array}$

Site

Left $\quad 44$ (41.3)

Molecular subtype

Right

$31(58.7)$

$\begin{array}{ll}\text { Her2 positive } & 21(28.0) \\ \text { Luminal-Her2 (-) } & 22(29.3) \\ \text { Luminal-Her2 positive } & 14(18.7) \\ \text { TNBC } & 18(24.0)\end{array}$

Clinical stage

T stage

N stage

T4

16 (21.3)

39 (52.0)

15 (20.0)

5 (6.7)

Tissue stage

N3

Ki67

$\begin{array}{ll}<14 \% & 18(24.0) \\ \geq 14 \% & 57(76.0)\end{array}$

pCR state

pCR $20(26.7)$

Non-pCR 55 (73.3) 
3. PD-L1 assessment: (1) PD-L1 staining in tumor cells and lymphocytes was first observed under low magnification $(\times 10)$; samples with any positive staining of tumor cells, lymphocyte membranes and/or lymphocyte cytoplasm were defined as PD-L1 positive. (2) The percentages of PD-L1positive cells among all tumor cells and lymphocyte regions were determined in three regions with abundant expression of PD-L1 (i.e., hotspots) under high magnification, and the mean value was obtained.

4. MVD and MPI: (1) First, blue fluorescence was selected to observe cell nuclei and locate the tumor area. Red fluorescence was then selected, and the area with the most abundant red staining (i.e., CD105-positive staining) in the tumor region was selected at low magnification $(\times 10)$ (hotspot) and photographed at high magnification $(\times 200)$. (2) Green fluorescence was selected and photographed. (3) Blue fluorescence was selected and photographed. (4) Photographs taken in the first 3 steps were merged. (5) A total of 3 hotspot regions were selected for photographing and image merging using the same method. (6) The MVD of CD105positive microvessels and the number of microvessels covered with NG2 were determined in each merged image. MPI =
Number of microvessels covered with NG2/Total number of microvessels * 100\%. Finally, the mean MPI was calculated.

5. Evaluation of sTILs and PD-L1 in lymph nodes: First, tumor cell-rich lymph nodes were selected under low magnification, and all indicators were then evaluated under high magnification according to the interpretation method for tumor tissues.

All sections were read twice, and the second reading was performed 2-4 weeks after the first reading. When differences of more than $10 \%$ were observed between the two readings, the sections were read again, and finally, the mean values were taken. For cases that achieved pCR, the TILs count and PD-L1 expression in the tumor bed was evaluated.

\section{Statistical Analysis}

All count data are expressed as the mean \pm standard deviation. Pearson's chi-square test or Fisher's exact test was used to compare categorical variables, and a rank sum test was used to compare numerical variables. Univariate and multivariate analyses were performed using binary logistic regression analysis, and the odds ratio (OR), 95\% confidence interval (95\% CI) and

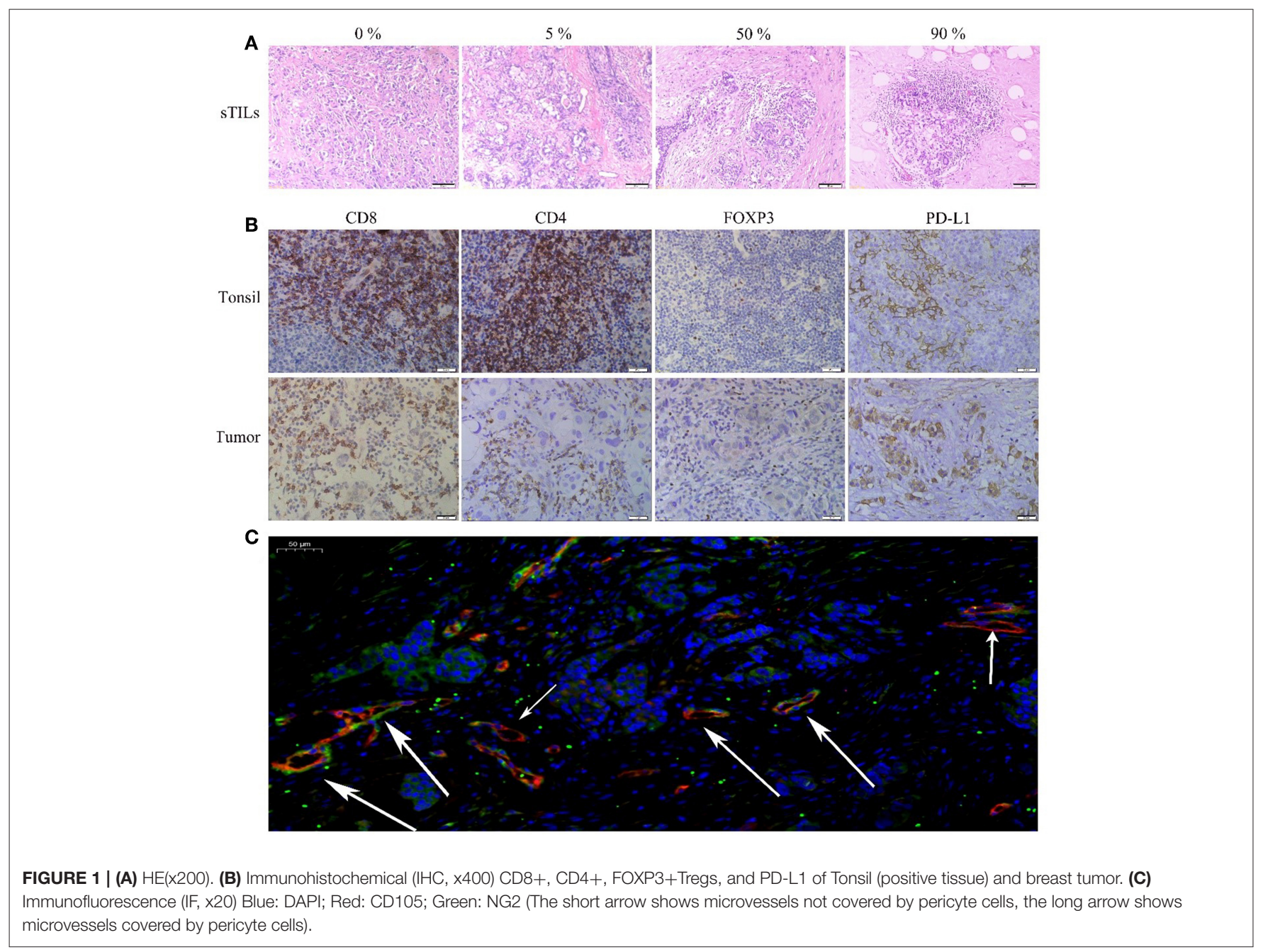




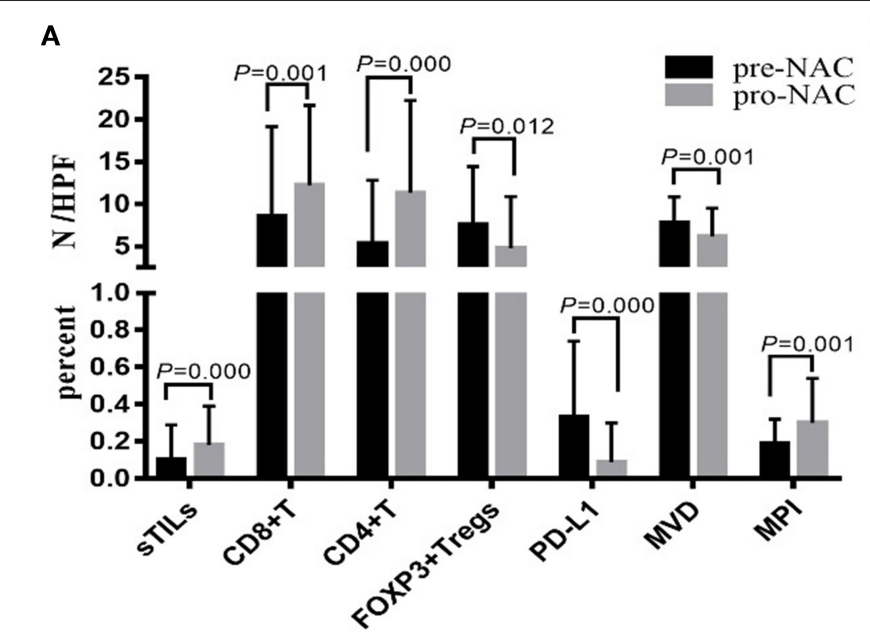

C

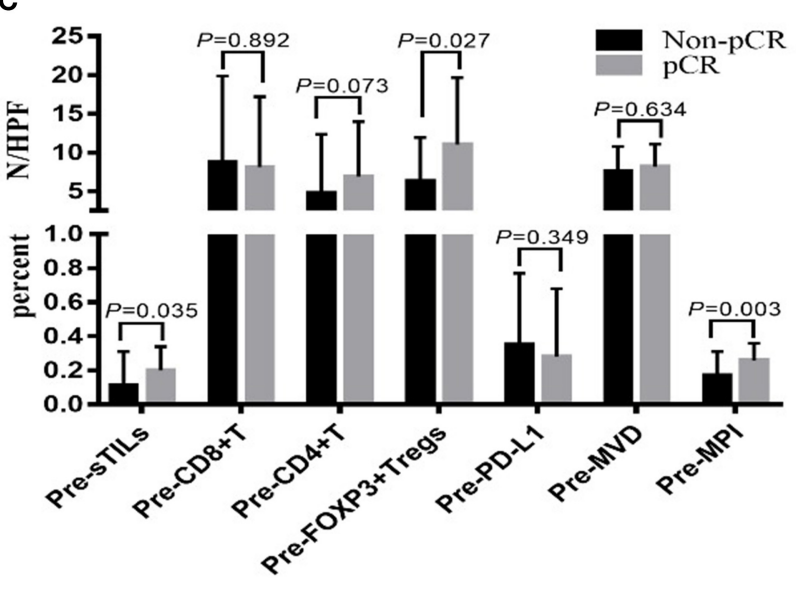

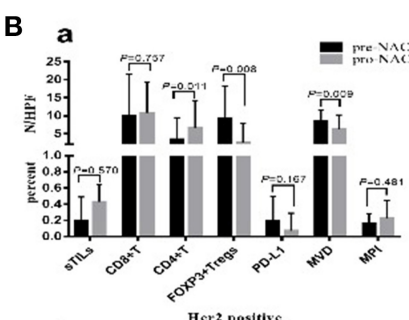

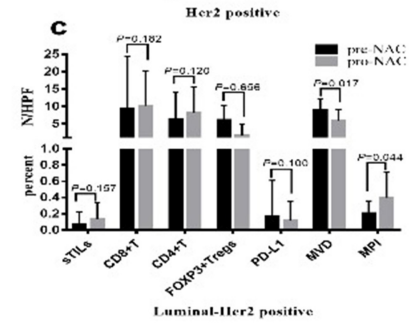

D
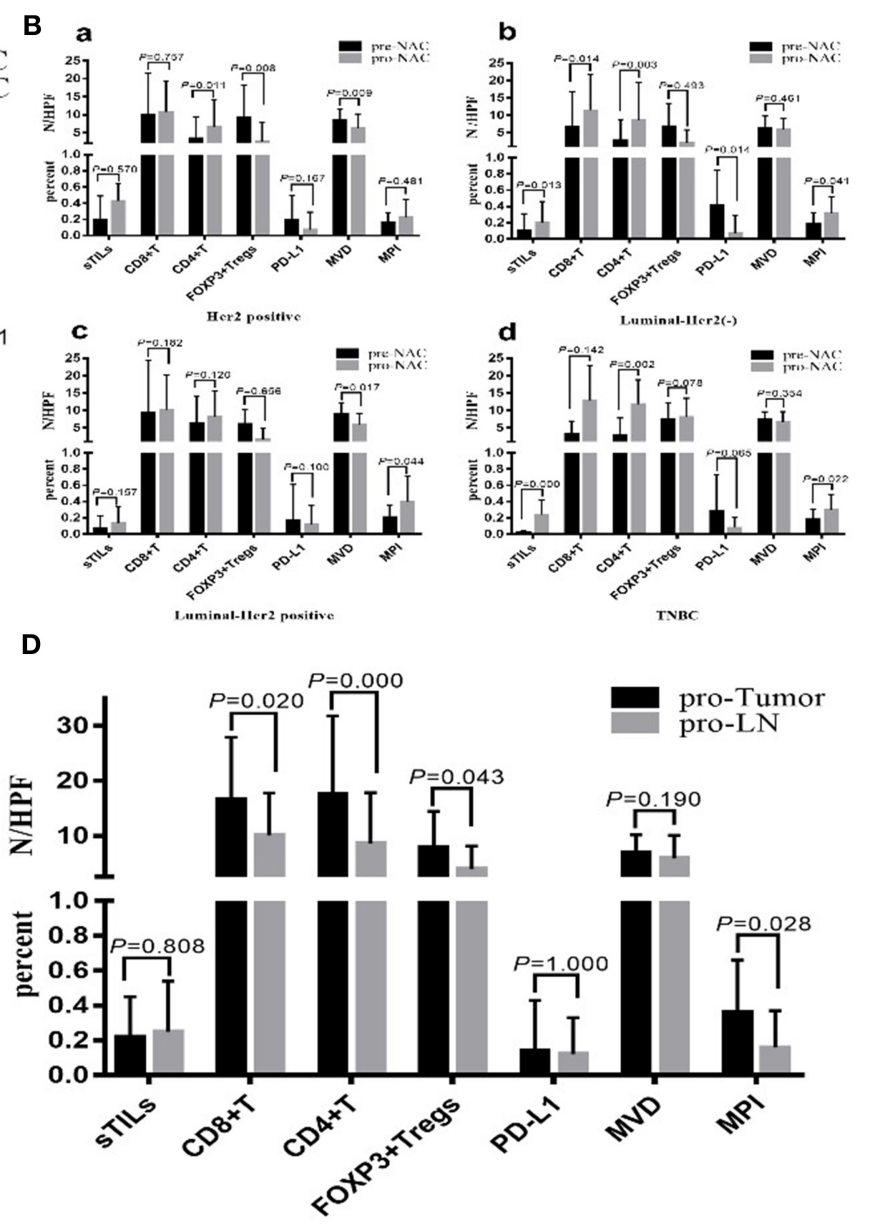

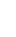

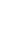


CD8 + T cells, CD4+ T cells, and FOXP3 + Tregs and the PDL1 expression, MVD, and MPI values before and after NAC, our study found that after NAC, sTILs, CD8 + T cells, CD4 + T cells, and MPI were increased in tumor tissue, and FOXP3+ Tregs, PD-L1 expression, and MVD were decreased in tumor tissue (Figure 2A). Comparison of patients with different molecular subtypes of $\mathrm{BC}$ revealed that after NAC, the Her2-positive patients showed significantly enhanced infiltration of CD4+ T cells $(P=0.011)$ and significantly reduced FOXP3+ Tregs and $\operatorname{MVD}\left(P_{\text {FOXP3 }+ \text { Tregs }}=0.008, P_{\mathrm{MVD}}=0.009\right)$. However, there was no significant difference in the total number of sTILs and CD8+ T cells, MPI, and PD-L1 expression before and after NAC (Figure 2B). After NAC, patients with luminal/HER2-negative $\mathrm{BC}$ showed a significant increase in sTILs, CD8+ T cells, CD4+ $\mathrm{T}$ cells and MPI $(P<0.05)$; a significant decrease in PDL1 expression $(P=0.041)$; and a non-significant decrease in MVD and FOXP3+ Tregs (Figure 2B). After NAC, patients with luminal/HER2-positive BC presented a significant increase in MPI $(P=0.044)$, a significant decrease in MVD $(P=0.017)$, and a non-significant change in the number of sTILs, CD8+ $\mathrm{T}$ cells, CD4+ $\mathrm{T}$ cells, and FOXP3+ Tregs and in PD-L1 expression (Figure 2B). After NAC, patients with TNBC showed a significant increase in sTILs, CD8 $+\mathrm{T}$ cells, CD4 $+\mathrm{T}$ cells and MPI $(P<0.05)$ (Figure 2B).

Baseline sTILs and FOXP3+ Tregs and MPI in tumor tissues from $\mathrm{BC}$ patients in the pCR group were higher than those in the Non-pCR group $\left(P_{\text {sTILs }}=0.035, P_{\text {Tregs }}=0.027, P_{\text {MPI }}=0.005\right)$. There was no significant difference in CD8 $+\mathrm{T}$ cells, CD4+ T cells, PD-L1 expression, and MVD between the patients in the pCR and Non-pCR groups (Figure 2C).

From comparison of the post-NAC changes in sTILs, CD8+ T cells, CD4+ T cells, FOXP3 + Tregs, PD-L1 expression, MVD, and MPI between BC patients in the pCR and Non-pCR groups, the percentage of patients with enhanced sTILs, CD8+ T cells, and CD4+ T cells infiltration in the pCR group was found to be significantly higher than that in the Non-pCR group $(P<0.05)$, while the percentage of patients with reduced FOXP3 + Tregs in the pCR group was significantly higher than that in the Non-pCR group $(P=0.000)$ (Table 3$)$.

\section{sTILs, CD8+ T Cells, CD4+ T Cells, and FOXP3+ Tregs Counts; PD-L1 Expression; MVD; and MPI in Primary Tumors and Metastatic Lymph Nodes After NAC}

Among the 55 patients with $\mathrm{BC}$ in the non-pCR group, 25 cases were confirmed by postoperative pathology to have lymph node metastasis. For four patients among the 25 cases, only small specimens, which could not be further divided due to the small amount of metastatic cancer components, could be obtained from postoperative metastatic lymph nodes. Therefore, in this study, the postoperative sTILs count, PD-L1 expression, MVD and MPI in primary tumors and metastatic lymph nodes in 21 patients were compared (Figure 2D). The results showed that CD8+ T cells, CD4+ T cells, FOXP3 + Tregs and MPI in primary tumors were all higher than those in metastatic lymph nodes $\left(P_{\mathrm{CD} 8+}=0.020, P_{\mathrm{CD} 4+}=0.000, P_{\mathrm{FOXP} 3+\text { Tregs }}=0.043, P_{\mathrm{MPI}}\right.$
TABLE 3 | Changes of sTILs, PD-L1, MVD, and MPI in non-pCR group and pCR group after NAC.

\begin{tabular}{|c|c|c|c|c|}
\hline Parameter & & $\begin{array}{l}\text { Non-pCR } \\
\text { group }\end{array}$ & pCR group & $P$ \\
\hline \multirow[t]{4}{*}{ sTILs change } & & & & 0.020 \\
\hline & Up & $34(61.8)$ & $19(95.0)$ & \\
\hline & Down & $14(25.5)$ & $1(5.0)$ & \\
\hline & Stable & $7(12.7)$ & $0(0.0)$ & \\
\hline \multirow[t]{4}{*}{ CD8+T change } & & & & 0.037 \\
\hline & Up & $33(60.0)$ & $18(90.0)$ & \\
\hline & Down & $14(25.5)$ & $2(10.0)$ & \\
\hline & Stable & $8(14.5)$ & $0(0.0)$ & \\
\hline \multirow[t]{4}{*}{ CD4+T change } & & & & 0.012 \\
\hline & Up & 35 (63.6) & $19(95.0)$ & \\
\hline & Down & $6(10.9)$ & $1(5.0)$ & \\
\hline & Stable & $14(25.5)$ & $0(0.0)$ & \\
\hline \multirow[t]{4}{*}{ FOXP3+Tregs change } & & & & 0.000 \\
\hline & Up & $25(45.5)$ & $0(0.0)$ & \\
\hline & Down & $22(40.0)$ & $17(85.0)$ & \\
\hline & Stable & $8(14.5)$ & $3(15.0)$ & \\
\hline \multirow[t]{4}{*}{ PD-L1 change } & & & & 0.627 \\
\hline & Up & 13 (23.6) & 7 (35.0) & \\
\hline & Down & $29(52.7)$ & $9(45.0)$ & \\
\hline & Stable & $13(23.6)$ & $4(20.0)$ & \\
\hline \multirow[t]{4}{*}{ MVD change } & & & & 0.478 \\
\hline & Up & $16(29.1)$ & $3(15.0)$ & \\
\hline & Down & $37(67.3)$ & $16(80.0)$ & \\
\hline & Stable & 2 (3.6) & $1(5.0)$ & \\
\hline \multirow[t]{4}{*}{ MPI change } & & & & 0.557 \\
\hline & Up & $36(65.5)$ & $16(80.0)$ & \\
\hline & Down & $16(29.1)$ & $4(20.0)$ & \\
\hline & Stable & $3(5.5)$ & $0(0.0)$ & \\
\hline
\end{tabular}

$=0.028$ ), while there were no significant differences in sTILs count, PD-L1 expression and MVD between primary tumors and metastatic lymph nodes.

\section{Univariate and Multivariate Analyses of pCR}

From the binary logistic univariate analysis of patient age, T stage, $\mathrm{N}$ stage, histological grade, Ki67, preNAC sTILs (Pre-sTILs), preNAC CD8 $+\mathrm{T}$ cells (Pre-CD8 $+\mathrm{T}$ cells), preNAC CD4 $+\mathrm{T}$ cells (Pre-CD4+ T cells), preNAC FOXP3 + Tregs (Pre-FOXP3+ Tregs), preNAC PD-L1 (Pre-PD-L1), preNAC MVD (Pre-MVD), and preNAC MPI (Pre-MPI), the Pre-FOXP3+ Tregs count and Pre-MPI were found to be correlated with pCR $(P<0.05)$ (Table 4). From the multivariate analysis of histological grades, preNAC Ki67, Pre-sTILs, Pre-CD8+ T cells, Pre-CD4+ T cells, Pre-FOXP3+ Tregs, Pre-PD-L1, Pre-MVD and Pre-MPI, the Pre-FOXP3+ Tregs count, Pre-MPI and Ki67 were found to be correlated with pCR $(P<0.05)$ (Table 4$)$.

We also analyzed the relationship between the baseline ratio of populations (Tregs/T cells) and pCR (Table 5), and the results 
TABLE 4 | Univariate and multivariate analysis of pCR after NAC.

\begin{tabular}{|c|c|c|c|c|c|c|}
\hline \multirow[b]{2}{*}{ Parameter } & \multicolumn{3}{|c|}{ Univariate analysis } & \multicolumn{3}{|c|}{ Multivariate analysis } \\
\hline & OR & $95 \% \mathrm{Cl}$ & $P$ & OR & $95 \% \mathrm{Cl}$ & $\boldsymbol{P}$ \\
\hline age & 1.028 & 0.968-1.092 & 0.362 & - & - & - \\
\hline T stage & 0.625 & $0.289-1.350$ & 0.232 & - & - & - \\
\hline N stage & 0.589 & $0.299-1.160$ & 0.126 & - & - & - \\
\hline Tissue stage & 1.968 & $0.439-2.134$ & 0.936 & 0.499 & $0.176-1.417$ & 0.192 \\
\hline Ki67 & 6.925 & $0.757-63.313$ & 0.087 & 28.600 & $1.262-648.302$ & 0.035 \\
\hline Pre-sTILs & 0.739 & $0.042-12.872$ & 0.836 & 2.220 & $0.014-357.134$ & 0.758 \\
\hline Pre-CD8+T & 0.994 & $0.946-1.045$ & 0.825 & 0.879 & $0.767-1.007$ & 0.062 \\
\hline Pre-CD4+T & 1.037 & $0.971-1.107$ & 0.278 & 1.081 & $0.969-1.205$ & 0.162 \\
\hline Pre-FOXP3+ & 1.109 & $1.019-1.206$ & 0.016 & 1.210 & $1.057-1.386$ & 0.006 \\
\hline Pre-PD-L1 & 0.618 & $0.901-1.261$ & 0.456 & 0.461 & $0.087-2.447$ & 0.363 \\
\hline Pre-MVD & 1.066 & $0.901-1.261$ & 0.456 & 0.927 & $0.730-1.177$ & 0.533 \\
\hline Pre-MPI & 273.009 & 3.583-20802.657 & 0.011 & 6275.715 & $18.587-2118883.492$ & 0.003 \\
\hline
\end{tabular}

TABLE 5 | Relationship between the baseline ratios of populations and pCR.

\begin{tabular}{lccc}
\hline Parameters & pCR group & Non-pCR group & $P$-value \\
\hline Foxp3+/CD8+ & $1.15 \pm 0.78$ & $0.34 \pm 0.52$ & 0.001 \\
Foxp3+/CD4+ & $1.64 \pm 1.58$ & $0.44 \pm 0.61$ & 0.001 \\
\hline
\end{tabular}

showed that both Foxp3+/CD8+ and Foxp3+/CD4+ had a higher ratio in $\mathrm{pCR}$ group than in non-pCR group.

\section{Survival Analysis}

By the time of data analysis (June 1,2019), the median follow-up time for the 75 patients with $\mathrm{BC}$ was $23.2 \mathrm{mo}(6.1-64.5 \mathrm{mo})$, the median follow-up time for the patients in the pCR group was 22.3 mo (9.9-61.4 mo), and the median follow-up time of the patients in the Non-pCR group was 24.9 mo (6.1-64.5 mo). During the follow-up period, 17 patients $(22.7 \%)$ had disease progression $(7$ cases of Her2-positive BC, 5 cases of luminal/HER2-positive BC, 2 cases of luminal/HER2-positive BC, 3 cases of TNBC); among them, 1 case was from the pCR group, and 16 cases were from the Non-pCR group. The data for survival analysis were immature and could not be used to calculate the median DFS of the patients. According to the preliminary data analysis, the DFS in the pCR group was significantly longer than that in the Non-pCR group $(P=0.049)$ (Figure 3A).

Subgroup analysis showed that after NAC, the DFS of BC patients with reduced FOXP3+ Tregs was significantly better than that of BC patients with increased FOXP3+ Tregs $(P=$ 0.029) (Figure 3E). Currently, no correlation was found between DFS and the changes in sTILs, CD8 + T cells, CD4+ T cells, PDL1 expression, MVD and MPI after NAC (Figures 3B-D,F-H).

The relationship between sTILs and their subtypes, PD-L1 expression, MVD and MPI in metastatic lymph nodes and the prognosis of BC patients after NAC was explored. The median values were used to divide patients into high-expression and low-expression groups. No significantly correlation was found between DFS and the total sTILs, FOXP3+ Tregs, CD8+ T cells and CD4+ T cells; PD-L1 expression; or MPI and MVD in BC patients $(P>0.05)$ (Figure 4).

\section{DISCUSSION}

This study showed that after BC patients underwent NAC, sTILs, CD8+ T cells, CD4+ T cells and MPI increased, and FOXP3+ Tregs, PD-L1 expression and MVD decreased. The increase in sTILs and the decrease in PD-L1 expression were consistent with the results of Pelekanou et al. (22). However, Pelekanou et al. did not classify TILs, while we analyzed the subtypes of BC and found that the reduction in FOXP3 + Tregs was most prominent in patients with Her2-positive BC. NAC changed the number of sTILs in the tumor bed. Whether this change is correlated with pCR and DFS of patients has rarely been studied. Our study found that the percentage of patients with increased sTILs, CD8 + T cells, and CD4+ T cells was significantly higher in the pCR group than the Non-pCR group, while the percentage of patients with decreased FOXP3 + Tregs was significantly higher in the pCR group than the Non-pCR group. Further analysis of DFS showed that a decrease in FOXP3+ Tregs was significantly correlated with DFS. These results suggest that the increase in sTILs, CD8 $+\mathrm{T}$ cells and CD4+ $\mathrm{T}$ cells and the reduction in FOXP3+ Tregs might be related to the efficacy of NAC in BC patients. If the changes in these factors in tumor tissue can be dynamically detected during NAC, they may become predictive factors for the efficacy of NAC for BC. We did not find the significant difference of TILs between HER-2 positive and HER2 negative $\mathrm{BC}$, although all HER-2 positive BC patients received Trastuzumab treatment that was related to TILs (23). More study should be developed to focus this topic.

In addition to TILs, the vascular normalization-related indicators MPI and MVD were also examined in the present study. The results showed that after NAC, MPI was increased in all BC subtypes, suggesting that NAC can promote tumor vessel normalization. Meanwhile, the reduction in MVD was consistent with the reduction in FOXP3 + Tregs and PD-L1 expression, 
A

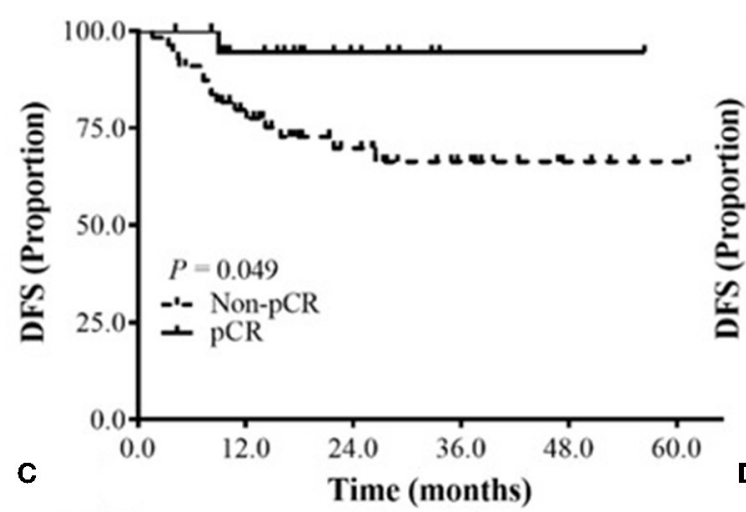

B
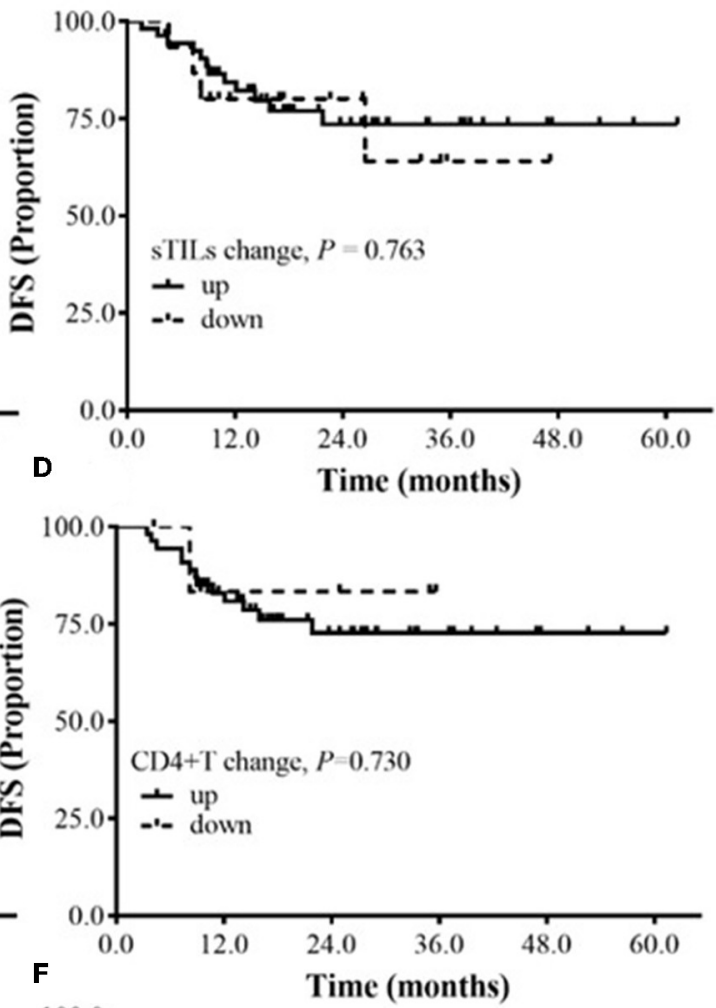

E

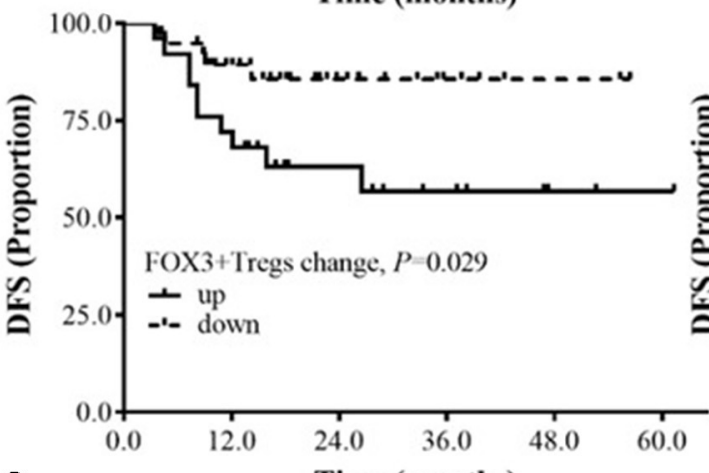

G

Time (months)

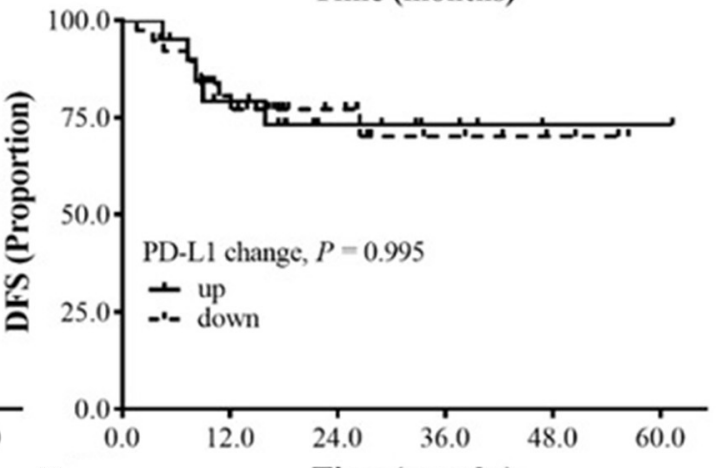

$\mathbf{H}$

Time (months)

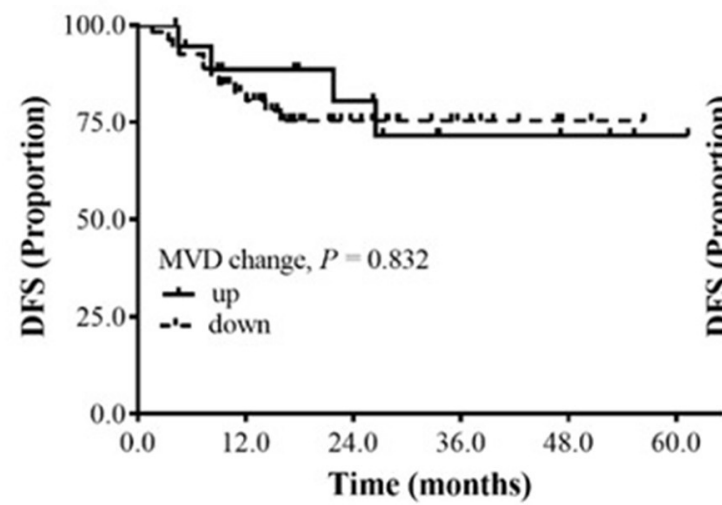

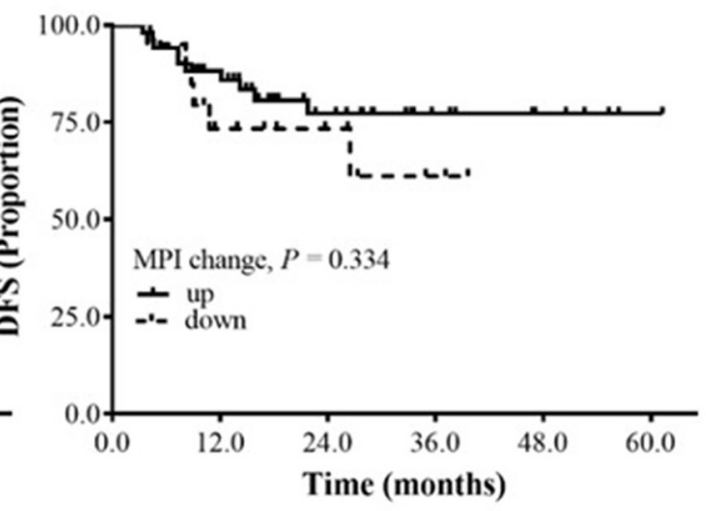

FIGURE 3 | The relationship between the changes of STILS, PD-L1, MVD, MPI, and DFS before and after NAC. (A) Survival analysis between pCR group and Non-pCR group. (B) Survival analysis of sTILs change. (C) Survival analysis of CD8+T change. (D) Survival analysis of CD4+T change. (E) Survival analysis of FOXP3+ Tregs change. (F) Survival analysis of PD-L1 change. (G) Survival analysis of MVD change. (H) Survival analysis of MPI change. 

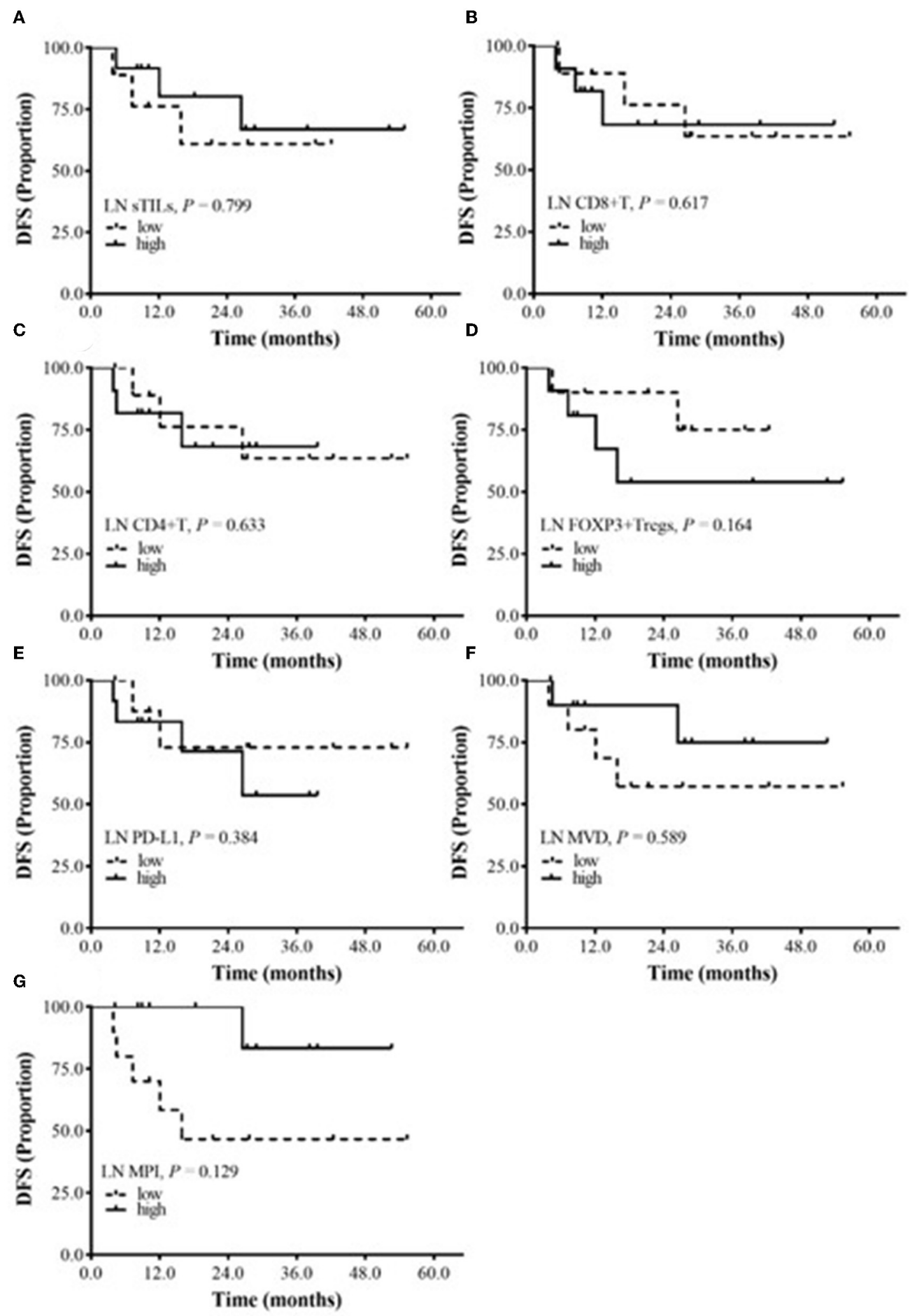

FIGURE 4 | The relationship between DFS and sTILs, PD-L1, MVD, and MPI in Metastatic lymph node (LN). (A) Survival analysis of LN sTILs. (B) Survival analysis of LN CD8+ T cells. (C) Survival analysis of LN CD4+ T cells. (D) Survival analysis of LN FOXP3+ Tregs. (E) Survival analysis of LN PD-L1. (F) Survival analysis of LN MVD. (G) Survival analysis of LN MPI. 
and the three may have synergistic effects. Tumor vessels often have abnormal structural and functional manifestations, such as a low MPI, an uneven basement membrane, and loose cell-to-cell connections. These abnormalities impair blood perfusion and drug delivery and increase the level of hypoxia in tumors and limit extravasation of $\mathrm{T}$ cells, especially cytotoxic $\mathrm{T}$ lymphocytes (CTLs) (24). Tumor vessel normalization enhances $\mathrm{T}$ cell infiltration and function. Tian et al. reported that effector CD4+ T cells supported vascular normalization and emphasized the interaction between blood vessels and $\mathrm{T}$ cells in cancer (25). Tolaney et al. studied the response of vascular density and normalization in $\mathrm{BC}$ patients to neoadjuvant bevacizumab and chemotherapy and found that a high baseline MVD in $\mathrm{BC}$ patients might be a necessary condition for bevacizumabinduced vascular normalization. Moreover, they found that an increased density of pericyte-covered microvessels was correlated with enhanced vascular function and oxygenation (20). The present study showed that MVD decreased and MPI increased after NAC, further confirming that the number of pericytes was inconsistent with the MVD. These results indirectly indicate that NAC promotes vascular normalization to some extent, enhances infiltration of $\mathrm{CD} 8+\mathrm{T}$ cells and $\mathrm{CD} 4+\mathrm{T}$ cells, and reduces infiltration of FOXP3+ Tregs.

Analysis of the correlation between sTILs in primary tumors and metastatic lymph nodes after NAC showed that CD8+ $\mathrm{T}$ cells, CD4+ T cells, FOXP3 + Tregs and MPI in primary tumors were higher than those in metastatic lymph nodes. From further analysis of the effects of them in the positive lymph nodes on the prognosis of BC patients, we found that the DFS of BC patients with low FOXP3 + Tregs and high MPI in the metastatic lymph nodes was better than that of patients with high FOXP3 + Tregs and low MPI; however, the difference was not statistically significant. This may be due to the small sample size in this study. Currently, no study has investigated sTILs and PD-L1 expression in primary tumors and metastatic lymph nodes after NAC in BC patients. The present study found that after NAC, the levels of CD8 $+\mathrm{T}$ cells, CD4+ T cells, FOXP3+ Tregs, and MPI in primary tumors were higher than those in metastatic lymph nodes, suggesting that the sensitivity of primary tumors to NAC may be different from the sensitivity of metastatic lymph nodes to NAC. These results need further validation. In addition, currently, few studies have assessed TILs in metastatic lymph nodes, and a standard assessment method is lacking. Kaewkangsadan et al. were the first to evaluate the abundance of TILs, Tregs (FOXP3+, CTLA4+) and NK cells in metastatic axillary lymph nodes (26). The evaluation method for TILs in metastatic lymph nodes still requires further exploration, and a unified standard is lacking for determining the TILs threshold, which requires the consensus of many researchers. In addition, evaluation of TILs in primary tumors and metastatic

\section{REFERENCES}

1. Global Burden of Disease Cancer C, Fitzmaurice C, Akinyemiju TF, Al Lami FH, Alam T, Alizadeh-Navaei R, et al. Global, regional, and national cancer incidence, mortality, years of life lost, years lived with disability, and disability-adjusted life-years for 29 cancer groups, 1990 to 2016: a systematic lymph nodes after NAC may provide a basis for more accurate prediction of patient prognosis and thus aid in selection of an optimal adjuvant treatment regimen.

This study is a relatively comprehensive study that explored the effect of NAC administered to BC patients on sTILs infiltration and PD-L1 expression in the TME of BC. However, this study still has drawbacks. First, the overall sample size of this study was small, leading to a low number of cases for each BC subtype, which might bias the results. Second, this study had a short follow-up period, with a median follow-up time of fewer than 5 years, and thus, the data for survival analysis were not yet mature. Third, the present study used core needle biopsy specimens before NAC to evaluate sTILs, PD-L1, MVD, and MPI, which might not be able to accurately reflect the those parameters in the entire tumor. In a previous study, Cha et al. (27) evaluated the consistency between TILs scores in tissue specimens from a core needle biopsy and TILs scores of resected BC specimens and found that the TILs scores of the core needle biopsy specimens were reliable in reflecting the TILs status of the entire surgically resected tumor.

\section{CONCLUSION}

The baseline sTILs, FOXP3+ Tregs, and MPI in breast tumors can be used as predictive factors for the efficacy of NAC for BC patients. NAC increases infiltration of cells with immunological effects and reduces infiltration of immunosuppressive FOXP3+ Tregs and PD-L1. Changes in FOXP3 + Tregs infiltration and MPI may be used as prognostic markers in BC patients.

\section{DATA AVAILABILITY STATEMENT}

The raw data supporting the conclusions of this manuscript will be made available by the authors, without undue reservation, to any qualified researcher.

\section{ETHICS STATEMENT}

The studies involving human participants were reviewed and approved by Huazhong University of Science and Technology Institutional Ethics Committee. The patients/participants provided their written informed consent to participate in this study.

\section{AUTHOR CONTRIBUTIONS}

QX analyzed the data and carried out the experiment. LY, TH, YC, and JW did data collection and structure determination. QW, $\mathrm{XN}$, and JC designed the study and revised the manuscript.

analysis for the global burden of disease study. JAMA Oncol. (2018) 4:1553-68 doi: 10.1200/JCO.2018.36.15_suppl.1568

2. Bray F, Ferlay J, Soerjomataram I, Siegel RL, Torre LA, Jemal A. Global cancer statistics 2018: GLOBOCAN estimates of incidence and mortality worldwide for 36 cancers in 185 countries. CA Cancer J Clin. (2018) 68:394-424. doi: $10.3322 /$ caac. 21492 
3. Chen W, Sun K, Zheng R, Zeng H, Zhang S, Xia C, et al. Cancer incidence and mortality in China, 2014. Chin J Cancer Res. (2018) 30:1-12. doi: 10.21147/j.issn.1000-9604.2018.01.01

4. Zitvogel L, Kepp O, Senovilla L, Menger L, Chaput N, Kroemer G. Immunogenic tumor cell death for optimal anticancer therapy: the calreticulin exposure pathway. Clin Cancer Res. (2010) 16:3100-4. doi: 10.1158/1078-0432.CCR-09-2891

5. Emens LA: Breast cancer immunotherapy: facts and hopes. Clin Cancer Res. (2018) 24:511-20. doi: 10.1158/1078-0432.CCR-16-3001

6. Hamy AS, Pierga JY, Sabaila A, Laas E, Bonsang-Kitzis H, Laurent C, et al. Stromal lymphocyte infiltration after neoadjuvant chemotherapy is associated with aggressive residual disease and lower disease-free survival in HER2-positive breast cancer. Ann Oncol. (2017) 28:2233-40. doi: 10.1093/annonc/mdx309

7. Pitt JM, Marabelle A, Eggermont A, Soria JC, Kroemer G, Zitvogel L. Targeting the tumor microenvironment: removing obstruction to anticancer immune responses and immunotherapy. Ann Oncol. (2016) 27:1482-92. doi: 10.1093/annonc/mdw168

8. Salgado R, Denkert C, Demaria S, Sirtaine N, Klauschen F, Pruneri G, et al. The evaluation of tumor-infiltrating lymphocytes (TILs) in breast cancer: recommendations by an International TILs Working Group 2014. Ann Oncol. (2015) 26:259-71. doi: 10.1093/annonc/mdu450

9. Loi S. Tumor-infiltrating lymphocytes, breast cancer subtypes and therapeutic efficacy. Oncoimmunology. (2013) 2:e24720. doi: 10.4161/onci. 24720

10. Denkert C, Loibl S, Noske A, Roller M, Muller BM, Komor M, et al. Tumor-associated lymphocytes as an independent predictor of response to neoadjuvant chemotherapy in breast cancer. J Clin Oncol. (2010) 28:105-13. doi: 10.1200/JCO.2009.23.7370

11. Loi S, Sirtaine N, Piette F, Salgado R, Viale G, Van Eenoo F, et al. Prognostic and predictive value of tumor-infiltrating lymphocytes in a phase III randomized adjuvant breast cancer trial in node-positive breast cancer comparing the addition of docetaxel to doxorubicin with doxorubicin-based chemotherapy: BIG 02-98. J Clin Oncol. (2013) 31:860-7. doi: 10.1200/JCO.2011.41.0902

12. Savas P, Salgado R, Denkert C, Sotiriou C, Darcy PK, Smyth MJ, et al. Clinical relevance of host immunity in breast cancer: from TILs to the clinic. Nat Rev Clin Oncol. (2016) 13:228-41. doi: 10.1038/nrclinonc.2015.215

13. Lee HJ, Seo J-Y, Ahn J-H, Ahn S-H, Gong G. Tumor-associated lymphocytes predict response to neoadjuvant chemotherapy in breast cancer patients. $J$ Breast Cancer. (2013) 16:32-9. doi: 10.4048/jbc.2013.16.1.32

14. Denkert C, von Minckwitz G, Darb-Esfahani S, Lederer B, Heppner BI, Weber KE, et al. Tumour-infiltrating lymphocytes and prognosis in different subtypes of breast cancer: a pooled analysis of 3771 patients treated with neoadjuvant therapy. Lancet Oncol. (2018) 19:40-50. doi: 10.1016/S1470-2045(17)3 0904-X

15. Dong H, Strome SE, Salomao DR, Tamura H, Hirano F, Flies DB, et al. Tumor-associated B7-H1 promotes T-cell apoptosis: a potential mechanism of immune evasion. Nat Med. (2002) 8:793-800. doi: 10.1038/nm730

16. Zhu X, Zhang Q, Wang D, Liu C, Han B, Yang JM. Expression of PD-L1 attenuates the positive impacts of high-level tumor-infiltrating lymphocytes on prognosis of triple-negative breast cancer. Cancer Biol Ther. (2019) 2019:1-8. doi: 10.1080/15384047.2019.1595282
17. Goel S, Wong AH, Jain RK. Vascular normalization as a therapeutic strategy for malignant and nonmalignant disease. Cold Spring Harb Perspect Med. (2012) 2:a006486. doi: 10.1101/cshperspect.a006486

18. Type $1 \mathrm{~T}$ helper cells promote tumor vessel normalization. Cancer Discovery. (2017) 7:548.542-8. doi: 10.1158/2159-8290.CD-RW2017-073

19. De Palma M, Jain RK. CD4(+) $\mathrm{T}$ cell activation and vascular normalization: two sides of the same coin? Immunity. (2017) 46:773-5. doi: 10.1016/j.immuni.2017.04.015

20. Tolaney SM, Boucher Y, Duda DG, Martin JD, Seano G, Ancukiewicz M, et al. Role of vascular density and normalization in response to neoadjuvant bevacizumab and chemotherapy in breast cancer patients. Proc Natl Acad Sci USA. (2015) 112:14325-30. doi: 10.1073/pnas.1518808112

21. Eberhard A, Kahlert S, Goede V, Hemmerlein B, Plate KH, Augustin HG. Heterogeneity of angiogenesis and blood vessel maturation in human tumors: implications for antiangiogenic tumor therapies. Cancer Res. (2000) 60:138893.

22. Pelekanou V, Carvajal-Hausdorf DE, Altan M, Wasserman B, CarvajalHausdorf C, Wimberly $\mathrm{H}$, et al. Effect of neoadjuvant chemotherapy on tumor-infiltrating lymphocytes and PD-L1 expression in breast cancer and its clinical significance. Breast Cancer Res. (2017) 19:91. doi: 10.1186/s13058-017-0884-8

23. Krasniqi E, Barchiesi G, Pizzuti L, Mazzotta M, Venuti A, MaugeriSacca $M$, et al. Immunotherapy in HER2-positive breast cancer: state of the art and future perspectives. J Hematol Oncol. (2019) 12:111. doi: 10.1186/s13045-019-0798-2

24. Jain RK. Antiangiogenesis strategies revisited: from starving tumors to alleviating hypoxia. Cancer Cell. (2014) 26:605-22. doi: 10.1016/j.ccell.2014.10.006

25. Tian L, Goldstein A, Wang H, Ching Lo H, Sun Kim I, Welte T, et al. Mutual regulation of tumour vessel normalization and immunostimulatory reprogramming. Nature. (2017) 544:250-4. doi: 10.1038/nature21724

26. Kaewkangsadan V, Verma C, Eremin JM, Cowley G, Ilyas M, Eremin O Tumour-draining axillary lymph nodes in patients with large and locally advanced breast cancers undergoing neoadjuvant chemotherapy (NAC): the crucial contribution of immune cells (effector, regulatory) and cytokines (Th1, Th2) to immune-mediated tumour cell death induced by NAC. BMC Cancer. (2018) 18:123. doi: 10.1186/s12885-018-4044-z

27. Cha YJ, Ahn SG, Bae SJ, Yoon CI, Seo J, Jung WH, et al. Comparison of tumor-infiltrating lymphocytes of breast cancer in core needle biopsies and resected specimens: a retrospective analysis. Breast Cancer Res Treat. (2018) 171:295-302. doi: 10.1007/s10549-018-4842-7

Conflict of Interest: The authors declare that the research was conducted in the absence of any commercial or financial relationships that could be construed as a potential conflict of interest.

Copyright (c) 2020 Wang, Xiang, Yu, Hu, Chen, Wang, Nie and Cheng. This is an open-access article distributed under the terms of the Creative Commons Attribution License (CC BY). The use, distribution or reproduction in other forums is permitted, provided the original author(s) and the copyright owner(s) are credited and that the original publication in this journal is cited, in accordance with accepted academic practice. No use, distribution or reproduction is permitted which does not comply with these terms. 